\title{
Primary malignant ectomesenchymoma of the orbit
}

\author{
Thomas H Matsko, Rodney A Schmidt, Ann H Milam, James C Orcutt
}

\begin{abstract}
Malignant ectomesenchymoma (MEM) is a rare soft tissue tumour believed to arise from a pluripotential migratory neural crest cell and composed of both a mesenchymal element (most often rhabdomyosarcoma) and a neuroectodermal element (often neuroblastoma). Reported sites of origin are the abdomen, perineum or scrotum, the extremities, the middle ear, nasopharynx, face, and neck. We report the first case of an orbital MEM, with a review of the 17 cases previously reported from other sites.
\end{abstract}

Among children and adolescents malignancies account for only $7-9 \%$ of orbital disease. ${ }^{12}$ The most common primary malignant orbital neoplasm in children is rhabdomyosarcoma, ${ }^{12}$ accounting for $26 \%$ of all orbital malignancies. Neuroblastomas are usually metastatic in children and are not commonly subjected to biopsy when they occur in the orbit because the primary site is already known. The reported incidence of orbital involvement derived from two pathology series is $1 \%,{ }^{12}$ which is probably an underestimate of the true clinical incidence.

Malignant ectomesenchymoma (MEM) is a rare and unusual tumour composed of both a mesenchymal element, most often rhabdomyosarcoma, and a neuroectodermal element, often neuroblastoma. ${ }^{34}$ MEM are believed to arise from pluripotential migratory neural crest cells; they have been reported in the abdomen, perineum and scrotum, in the extremities, and in the middle ear, nasopharynx, face, and neck.

We report the first case of an orbital MEM, to our knowledge, with a review of the 17 cases previously reported from other sites.

Department of

Ophthalmology

T H Matsko

A H Milam

J C Orcutt

Department of

Pathology, University of

Washington, Seattle USA

R A Schmidt

Correspondence to:

James C Orcutt, MD,

Jepartment of

Department of

Ophthalmology RJ-10,

University of Washington,

Seattle,

Accepted for publication

21 November 1991

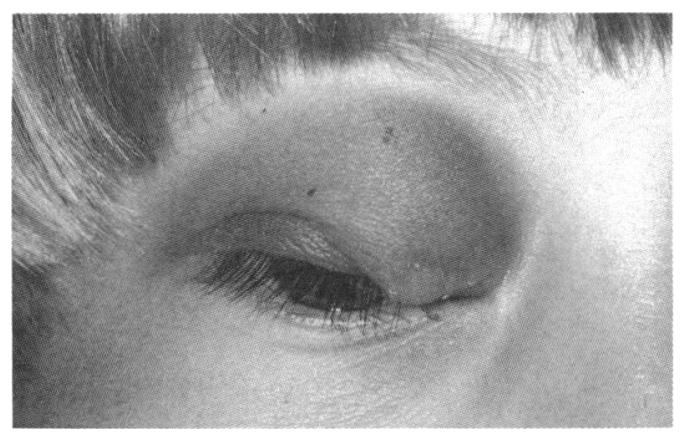

Figure 1 Ptosis induced by a right-sided superior nasal quadrant mass.

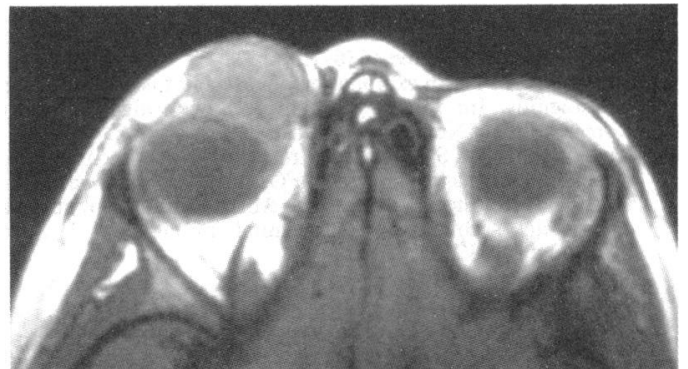

Figure 2A TI weighted MRI demonstrates low signal intensity of the tumour.

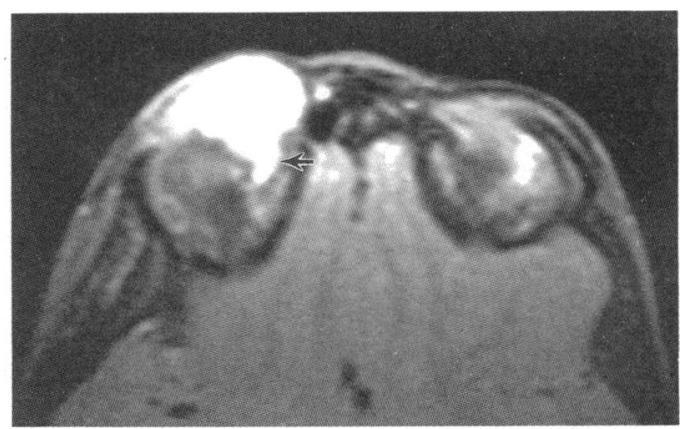

Figure 2B Gadolinium-enhanced T2 weighted MRI demonstrates enhancement of the mass. The tumour extends posteriorly in the superior nasal quadrant (arrow).

\section{Case history}

A 5 $1 \frac{1}{2}$ year old girl was referred to the University of Washington Eye Center with a 10-day history of right ptosis and an enlarging mass in the superior nasal quadrant of the right orbit. The patient denied pain or any change in vision. The parents stated the mass had grown rapidly over the past week. Past medical history and family history were unremarkable.

The visual acuity was $20 / 50$ on the right and $20 / 30$ on the left. Right ptosis $(7 \mathrm{~mm}$ ) was noted, and the right globe was displaced $2 \mathrm{~mm}$ temporally and $3 \mathrm{~mm}$ inferiorly (Fig 1). There was no proptosis. Supraduction of the right eye was limited. A $1.5 \mathrm{~cm}$ firm mass was palpable protruding from under the right orbital rim in the superior nasal quadrant.

Magnetic resonance imaging showed a well demarcated $1.8 \mathrm{~cm} \times 2.2 \mathrm{~cm} \times 2.2 \mathrm{~cm}$ soft tissue mass in the right superior nasal orbit. The mass was primarily in the preseptal space but extended posteriorly over the globe in the nasal quadrant to the equatorial region (Fig 2). The sinuses and nasal cavity were normal.

A trans-septal anterior orbital biopsy was performed with debulking of the tumour. 


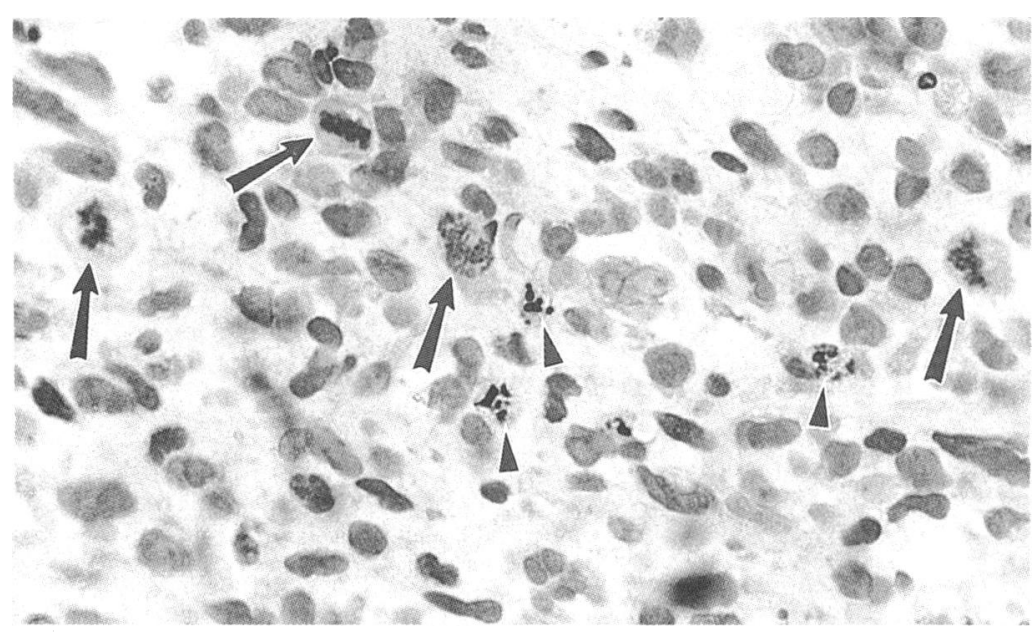

Figure 3 Loosely arranged round cells with scanty cytoplasm and hyperchromatic nuclei show marked pleomorphism with many mitotic figures (arrows). Some necrotic cells are present (arrowheads). (H-E, ×400.) times normal and $23 \%$ of cells in the DNA synthesis phase (S phase; normal $<5 \%$ ). The studies also indicated a small population of diploid cells.

Immunocytochemistry demonstrated two distinct, intermixed cell populations. One cell type was positive for desmin, vimentin, and muscle-specific actin. The other cell population was positive for chromogranin, nerve growth factor receptors, and neurofilaments. The specimen showed no reactivity with antibodies to lymphocytes, glial fibrillary acidic protein, carcinoembryonic antigen, or cytokeratins.

Electron microscopy demonstrated two cell populations, with regional segregation of cell types but some intermixing. One cell type consisted of closely apposed cells without cellular processes or basal laminae. These cells contained lipid and many mitochondria, as well as cytoplasmic filaments $5 \mathrm{~nm}$ and $15 \mathrm{~nm}$ diameter. Owing to the fixative used for electron microscopy, immunocytochemical confirmation that the $15 \mathrm{~nm}$ filaments were, in fact, myosin was not possible. No organised sarcomeres were identified, but foci of loose arrays of thick filaments were present in these cells (Fig 4). The other cell type included polygonal cells with numerous interdigitating cell processes. A few small intercellular junctions were present, but no desmosomes were found. These cells contained numerous mitochondria and $140-170 \mathrm{~nm}$ dense core neurosecretory-type granules (Fig 5).

The tumour was composed of loosely arranged embryonal mesenchymal cells of varying sizes and shapes. Most cells were round with scanty cytoplasm and hyperchromatic nuclei and some necrotic cells were present (Fig 3). There were many mitotic figures ( $>5$ per high power field), but no cross striations or rosettes were present (Fig 3).

Flow cytometry disclosed aneuploidy in $92 \%$ of the cells, with an average DNA content of $2 \cdot 8$

\section{CLINICAL EVALUATION}

Liver function tests, chest $x$ ray, chest and abdomen CT scans, cerebrospinal fluid cytology, liver-spleen scan, urinary catecholamines, and bone marrow were normal. The patient was treated according to the Intergroup Rhabdomyosarcoma Study III (IRS-III) regimen

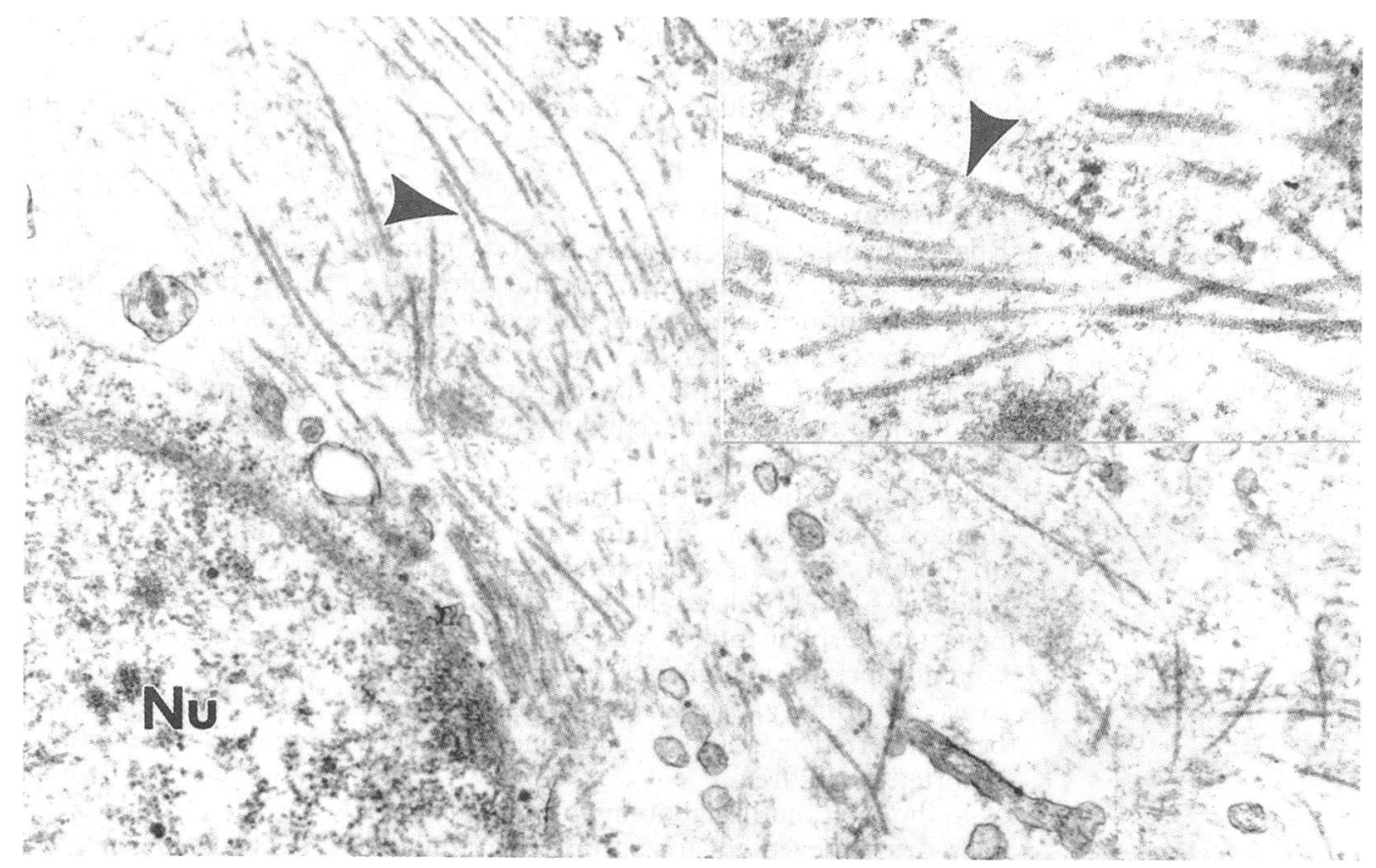

Figure 4 Electron micrograph. An array of $15 \mathrm{~nm}$ flaments (arrows), corresponds in size to myosin, but no sacromeres are observed ( $\times 30$ 000.) INSET: Higher magnification of myosin filaments (arrow.) $(\times 85000$. 


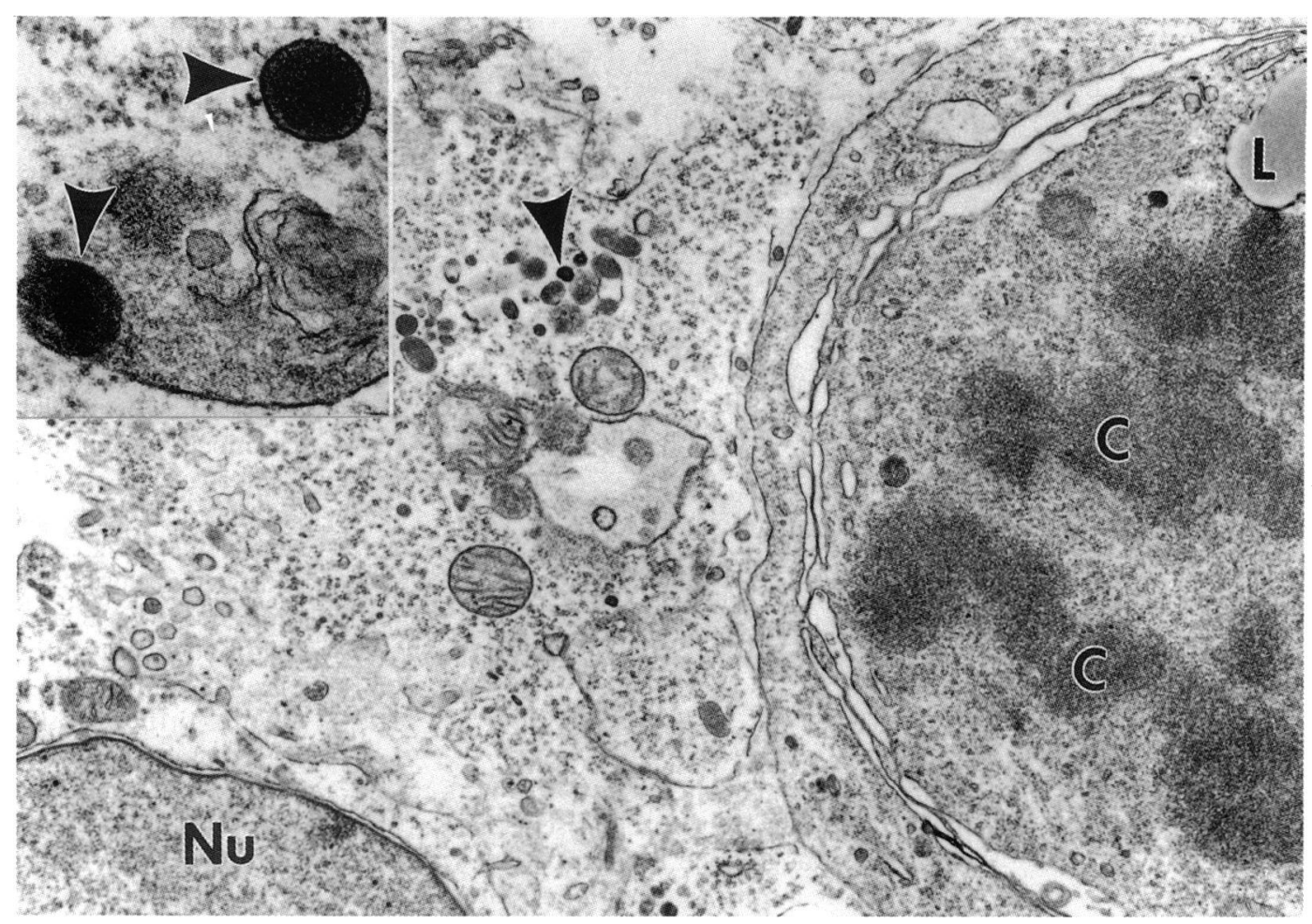

Figure 5 Electron micrograph. 140-170 nm dense-core neurosecretory granules are typical of neuroblastoma (arrows). ( $\times 8500$.) $C=$ dense chromosomes in a mitotic cell. $L=$ cytoplasmic lipid droplet. INSET: Higher magnification of dense-core granules (arrows). (85000.)

32 for rhabdomyosarcoma. Regimen 32 includes an initial course of 5 days of dactinomycin followed in 2 weeks by local radiation therapy and subsequent alternating courses of dactinomycin and vincristine. The patient's tumour grew rapidly during the dactinomycin treatment, so she was treated with $45 \mathrm{~Gy}$ of $x$-ray radiation in $1.8 \mathrm{~Gy}$ fractions to the tumour site beginning 10 days after initiation of chemotherapy. The tumour resolved, and the patient is well without recurrence or seconday tumour 18 months following completion of treatment. Follow-up magnetic resonance scans have not shown tumour regrowth. Her ptosis resolved and her vision improved to $20 / 25$ on the right.

\section{Discussion}

Rhabdomyosarcoma is the most common primary orbital malignancy ${ }^{256}$ and the third most common orbital lesion in children, ${ }^{2}$ typically presenting as a rapidly growing orbital mass, most commonly in the superior orbit. One histological hallmark of rhabdomyosarcoma is the presence of cytoplasmic cross striations, representing attempted sarcomere formation, though cross striations are found in only $50-60 \%$ of tumours and are not required for correct diagnosis. ${ }^{56}$ Neuroblastoma is the most common metastatic orbital tumour and the second most common orbital malignancy in children. ${ }^{2}$ Two cases of primary orbital neuroblastoma have been reported in adults, ${ }^{78}$ but none have been reported in children.

In the orbit, both rhabdomyoblasts and neural cells are believed to arise from pluripotential cells derived from migratory neural crest. ${ }^{579}$ In the developing embryo almost all connective tissue elements in the globe and orbit arise from cranial neural crest cells, perhaps correlated with lack of paraxial somites in the head and neck region. ${ }^{10-12}$ After migration into the orbit the neural crest cells undergo either mesenchymal or neuroectodermal differentiation, both of which are present in this tumour, which is accordingly termed 'ectomesenchymoma' or 'mesectodermoma'.

The tumour in this patient had intermixed mesenchymal and neuroectodermal components, as demonstrated by immunocytochemistry and electron microscopy. One type included cells that were positive for markers of skeletal muscle differentiation including desmin, vimentin, and muscle-specific actin. Although cross striations were not visible by light microscopy, electron microscopy demonstrated cytoplasmic filaments consistent with $15 \mathrm{~nm}$ myosin filaments. Myosin filaments occur primarily in striated muscle but are occasionally noted in smooth muscle cells. Myosin filaments in addition to coexpression of vimentin and desmin are diagnostic of rhabdomyosarcoma. ${ }^{6}$ The other tumour component was composed of cells that were positive for markers of neuroendocrine differentiation, including chromogranin, neurofilaments, and nerve growth factor receptors. Dense core secretory granules (140-170 nm diameter) and intertwining cell processes were observed ultrastructurally, typical of neuroblastoma and aesthesioneuroblastoma. These neurosecretory granules were significantly smaller than granules found in other neuroectodermal tumours such as carcinoid and paraganglioma.

The good general health of the patient and the normal results of physical, laboratory, and radiological examinations rule out meta- 
static neuroblastoma, aesthesioneuroblastoma, Ewing's sarcoma, and Wilms's tumour, and argue for the orbital primacy of this tumour. The fact that the two components of this patient's tumour were intermixed makes a so-called 'collision tumour' (two simultaneous primaries) less likely. In addition, flow cytometry demonstrated a single aneuploid population in this patient's tumour. Flow cytometry also showed that a high proportion of the tumour cells were in S-phase, consistent with the high mitotic rate observed by light microscopy.

MEM is a rare tumor, with 17 reported cases ${ }^{34}$ plus this case of the first orbital MEM, to our knowledge. MEM usually presents in infancy (average age 17 months, range $0-66$ months), but five cases have been reported in adults (average age 30 years, range 20-49 years). Various soft tissue sites have been involved: 10 cases occurred in the abdomen, perineum, or scrotum; two occurred in the extremities; six occurred in the head and neck region. Fifteen of the 18 cases contained rhabdomyosarcoma as the mesenchymal component and six of the 18 contained neuroblastoma as the neurosecretory component. At last follow-up eight patients (44\%) were alive without tumour (mean of 38 months, range of 6-144 months), and six had died (mean of 14.5 months after presentation, range of 6-40 months). Four patients were lost to follow-up, one with known local recurrence and another with metastases. Tumour dissemination has occurred by direct extension, haematogenous spread to the lungs, intraperitoneal spread, and metastases to lymph nodes. MEM is a highly malignant tumour and should be treated aggressively.

Successful treatment of MEM had been achieved by resection and chemotherapy, with or without radiation therapy to the tumour site. Given that rhabdomyosarcoma is a frequent malignant component of these tumours, it has been suggested that a chemotherapy protocol recommended for rhabdomyosarcoma would be a logical approach. ${ }^{3}$ Our patient was treated according to an IRS-III protocol. She responded well and is tumour-free with normal vision 18 months after presentation. Based on this case and reports in the literature, the appropriate treatment of MEM is maximal resection compatible with retention of function, combined with a rhabdomyosarcoma protocol of chemotherapy and radiation.

This work was supported in part by EY01311, EY01730, and by an award from Research to Prevent Blindness, Inc. Dr Milam is a senior scholar of RPB. Some facilities were supported by the Washington and Northern Idaho Lions' Sight Conservation Foundation, the Chatlos Foundation Inc, and the US Retinitis Pigmentosa Foundation, Inc.

1 Rootman J. Frequency and differential diagnosis of orbital disease. In: Rootman J, ed. Disease of the orbit: a multidisciplinary approach. Philadelphia: Lippincott, 1988: 119 39.

2 Iliff WJ, Green WR. Orbital tumors in children. In: Jakobiec FA, ed. Ocular and adnexal tumors. Birmingham: Aesculapius, 1978: 669-84.

3 Kawamoto EH, Weidner N, Agostini RM, Jaffe R. Malignant ectomesenchymoma of soft tissue: report of two cases and review of the literature. Cancer 1987; 59: 1791-802.

4 Kasantikul V, Shuangshoti S, Cutchavaree A, Bunyaphiphat P. Parapharyngeal malignant ectomesenchymoma: combined malignant fibrous histiocytoma and primitive combined malignant fibrous histiocytoma and primitive neuroectodermal tumour with ne
Laryngol Otol 1987; 101: 508-15.

5 Jakobiec FA, Font RL. Mesenchymal tumors. In: Spencer WH, ed. Ophthalmic pathology: an atlas and textbook. 3rd ed. Philadelphia: Saunders, 1986: 2554-66.

6 Knowles DM, Jakobiec FA, Potter HD, Jones IS. The diagnosis and treatment of rhabdomyosarcoma of the orbit. In: Jakobiec FA, ed. Ocular and adnexal tumors. Birmingham: Aesculapius, 1978: 708-26.

7 Bullock JD, Goldberg SH, Rakes SM, et al. Primary orbital neuroblastoma. Arch Ophthalmol 1989; 107: 1031-3.

8 Jakobiec FA, Klepach GL, Crissman JD, Spoor TC. Primary differentiated neuroblastoma of the orbit. Ophthalmology 1987; 94: 255-66.

9 Jakobiec FA, Iwamoto T. Ocular adnexa: introduction to lids, conjunctiva, and orbit. In: Jakobiec FA, ed. Ocular anatomy, embryology and teratology. Philadelphia: Harper and Row, 1982: 710 .

10 Johnson MC, Noden DM, Hazelton RD, et al. Origins of avian ocular and periocular tissues. Exp Eye Res 1979; 29: 27-43.

11 Noden DM. Periocular mesenchyme: neural crest and mesodermal interactions. In: Jakobiec FA, ed. Ocular anatomy, embryology and teratology. Philadelphia: Harper and Row, 1986: 97-119.

12 Jakobiec FA, Font RL. Orbit. In: Spencer WB, ed. Ophthalmic pathology: an atlas and textbook. 3rd ed. Philadelphia: Saunders, 1986: 2461-76. 